\title{
Study on Key Technologies in Product Lifecycle Data Management System for Spindle Bearings of Numerically Controlled Machine Tool
}

\author{
Yong CUI, a , Jishun $\mathrm{LI}^{2, \mathrm{~b}}$,Xin $\mathrm{SUI}^{3, \mathrm{C}}$ and Wei MA ${ }^{4, \mathrm{~d}}$ \\ ${ }^{1}$ Henan University of Science and Technology \\ Luoyang 471003, P.R. China \\ ${ }^{2}$ Henan Key Laboratory for Machinery Design and Transmission System \\ Henan University of Science and Technology \\ Luoyang 471003, P.R. China \\ ${ }^{3}$ Henan Key Laboratory for Machinery Design and Transmission System \\ Henan University of Science and Technology \\ Luoyang 471003, P.R. China \\ ${ }^{4}$ Henan Key Laboratory for Machinery Design and Transmission System \\ Henan University of Science and Technology \\ Luoyang 471003, P.R. China

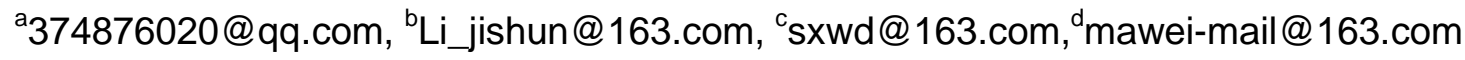

Keywords: Machine Tool Bearings; Production Lifecycle Management; Data Model; Integration Technology

\begin{abstract}
In order to fulfil the lifecycle data management for the spindle bearings of CNC machine tool, this paper firstly presented a bearings-lifecycle-oriented data management model, in which the bearings' code re-factoring methods and a ontology-based entity relationship (E-R) model are discussed in detail. Secondly, the key technologies of the data management system that utilized the presented data models, including the architecture, database design and integration schemas are described as well. Finally, a component-based system framework is presented to solve the heterogeneous problem in the process of XML-based bearing data extraction, transformation, cleaning and loading. An application demo will also be given to demonstrate the unity of management, integration, tracking and feedback for the lifecycle data management for spindle bearing.
\end{abstract}

\section{Introduction}

As the key components of machine devices, the spindle bearing and its quality has vital influence on main engine. The spindle bearing for high speed precision numerical control machine tool is an important component and has directly affect on the precision, rigidity, life and reliability of the machine tool. Hereby, data management system for spindle bearing that manages the whole lifecycle information of a series of spindle bearings for numerical control machine tool during its whole process such as development, production, application, operation and service is necessary to be fulfilled by all information technology means.

At present, there are many achievements about bearing design, manufacture, utilization, maintenance, abandonment and recycle. Yang Xian-qi et al. [1] adopted Product Data Management (PDM) to manage bearing parameters' data and implemented the functions such data storage, classify, retrieval and extraction. Ma Ming-xu [3] established the model of production lifecycle and solved the problem as the incomplete, 
inconsistent, and poor extensibility to express production. Kiviluoma R.et al. [3] has developed bridge load bearing evaluation system by using of network tool. Freire, Luís M.R., De Brito, Jorge and Correia, João R. [4] developed bridge bearing management system, which encompassed the development of a classification system of bridge support bearings, their anomalies and possible causes, inspection and diagnosis methods, and maintenance and rehabilitation techniques, linked by appropriately validated correlation matrixes. The existing research results do not involve the bearings' lifecycle data management. So the whole lifecycle data management system for the spindle bearing of numerical control machine tool is necessary to provide an information platform for the design, development, manufacture, utilization, maintenance, abandonment and recycle of high speed precision bearings.

\section{The Total Design for Data Management System for Spindle Bearings of Numerically Controlled Machine Tool}

Design of the BLDMS's Functions. The system requirements analysis guides the development and design of system functions. From the lifecycle angle, the BLDMS can be divided into six major functional modules, such as design information management, production information management, running information management, fault information management, maintenance information management, abandonment and recycle information management. Besides, some auxiliary functions will be developed to support system, such as system management and basic data management. Based on the various types of the managed information, the function modules are divided into 30+ more sub modules. This classification of functions is helpful for different person to supervise and control the bearings' data in different stages of lifecycle.

The system management mainly refers to information management and event management of operators. The "basic data management" mainly refers to the relatively stable data information. The "help information" refers to the vital information about the various stages of bearings. The design information management mainly refers to the "add", "delete" and "modify" operations for bearings parameters and other relevant information which are generated during the design process of spindle bearings. "Manufacturing information" mainly refers to the operation of basic information generated in production process, the quality information and static data, as well as the bearings' dynamic data of SPC. The "operation and maintenance information" refers to the static data before running, the dynamic data information after operation and the real-time monitoring data. The "fault data management" refers to the recording for the information for all breakdown bearings. "Scrap recycling information" refers to the recording of the data and parameters for normal/abnormal scrapped bearings that are no longer in service and needed to be recycled. The detailed structure and content for BLDMS functions is shown in fig1 as follows.

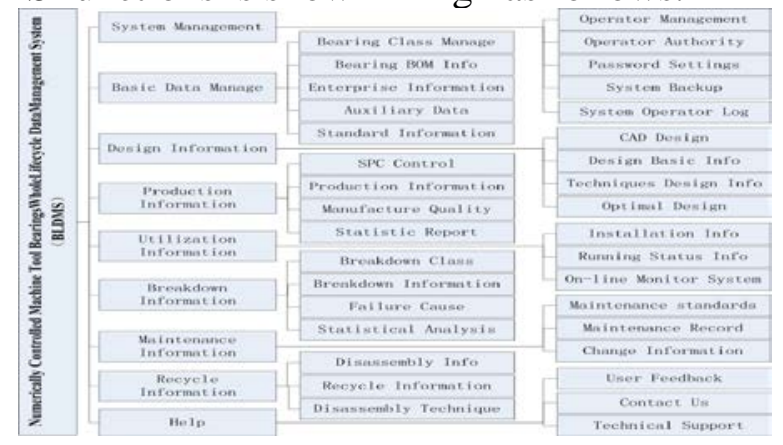

Fig.1. The system functions design for BLDMS 


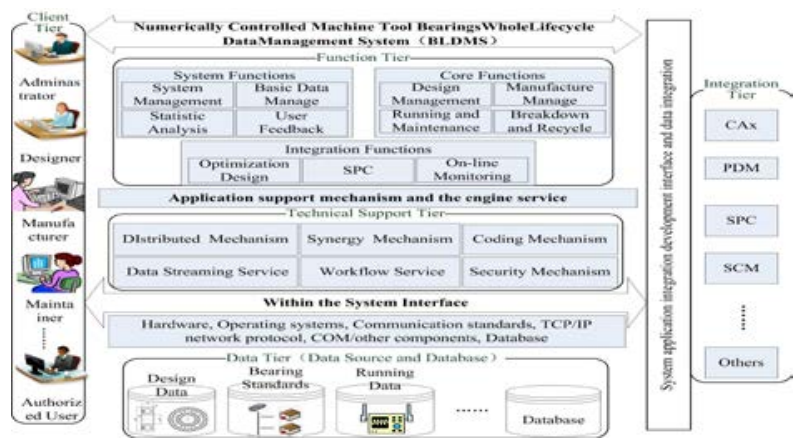

Fig.2. The architecture for BLDMS

Architecture of BLDMS.The system architecture is the basic construction of the entire system development and implementation. The architecture is divided into the client layer, function layer, technical support layer, integration layer and data layer.

The client layer mainly relates to the clients who operate the BLDMS. The clients may manage the bearing parameters data, life information and other knowledge resources for data extraction and analysis.

The function layer contains three parts: system function, core function, integration function. According to the requirements of system, each part has different contents.

The technical support layer refers to the general software and hardware technology, network protocol and related components to support system implementation.

The integration layer mainly integrates the existing achievements data and software. It can make full use of existing data resources, reduce the collection and acquisitions of data. This layer realizes the information integration, keeping the integrity of structure and function structure of information system.

The data layer stands for connection management of data source and database. The data source shows the diversity of data type so that it is required to find a way to extract data. The database is used to storage the data of design parameters, drawings, documents and other forms about bearings.

The system architecture is shown in fig 2.

As well known, a large amount of bearings' data is generating along with the bearing enterprise working which is stored in different systems. Though enterprises want to use these data quickly and conveniently in BLDMS, it is impossible to input these data by human works for the large time consuming, and even these data is isomers. Furthermore, because the bearing types are multiplex and complexity, Chinese standard or national standard is not satisfying the unique identification for bearings codes. Here leaves two questions to solve: the one is the heterogeneous data which express in structure, syntax and semantic aspects. Another is bearings codes. In order to solve these problems, this paper put forward metadata modeling and codes refactor.

The Design of Bearings Codes Refactor.The refactor of bearings codes is designed especially for manufacture stages, service stages and recycling stages, besides the design phase. The refactor principle adopts the way of bonding standard bearings type and production batch and product order which is based on GB/T271-1997 and GB/T272-1993. The angular contact ball bearing type of 7012AC is taken as an example to shown in table 1.

TABLE I. Codes Refactoring for Rolling Bearings’ Manufacture and Service

\begin{tabular}{c|c|c|c}
\hline \multirow{2}{*}{ Eeding Rule } & Code1 & Code 2 & Code 3 \\
\cline { 2 - 4 } Example & Products Standard & Production batch & Product order \\
\hline & $7012 \mathrm{AC}$ & 20150101 & 100 \\
\hline
\end{tabular}

As can be seen in table 1 , the new unique identification is 7012AC-20150101-100. Here the "-" is separator means that production batch is 20150101and product order is the hundredth. Thus, it is convenient to design manufacture and running data tables, and efficient to store and recognize data. 
Ontology-Based Metadata E-R Modeling .According to the characteristics of NC machine tool bearings, five kinds of rolling bearings are taken as the research object, such as angular contact ball bearings, tapered roller bearings, double row cylindrical roller bearings, angular contact thrust ball bearings, thrust roller bearings. The parameters of bearings are considered as the fields of data tables and are divided into different tables based on purpose, nature or characteristics, such as size, precision, parts test, et al. Therefore, the modeling method to design the E-R data model will be used. It not only is the precondition of system database, but also provides plenty of mapping space for multiple, heterogeneous data. The ontology-based E-R data model of system is shown in fig 3.

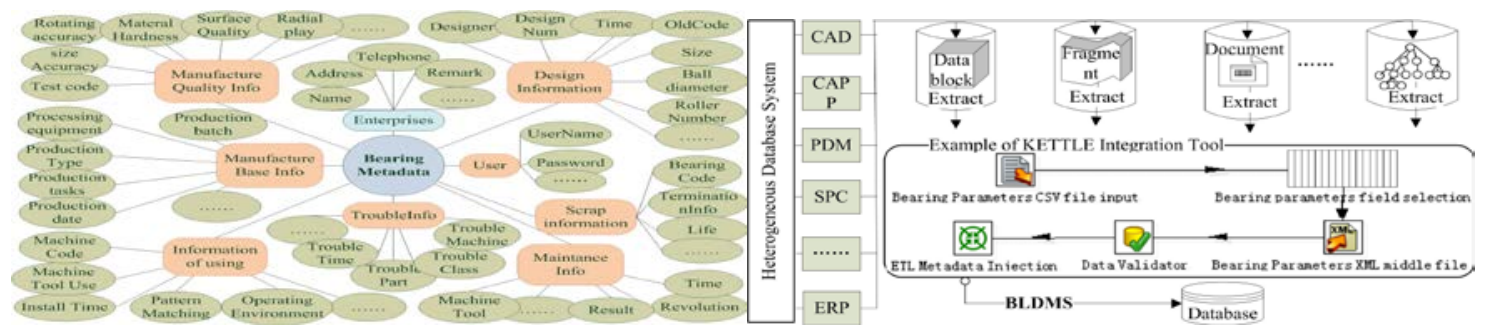

Fig.3. ontology-based E-R metadata model Fig.4 The data integration mechanism

\section{The Integration Technology}

The Application Integration Technology.In order to realize the integration of application systems, industry and academic circles put forward many kinds of technical scheme to support the enterprise application integration (EAI). But as a result of the heterogeneity and confidentiality of enterprise internal application system, the construction of information integration system has the certain difficulty. Therefore, standard for the exchange of product model data (STEP) is proposed. STEP standard adopts integration method of combining characteristic format files and sharing database, which aiming at the realization of product data exchange and sharing. At a certain extent, it realizes information integration between CAD, CAPP and CAM, etc., reduces the cost of the product life cycle information exchange and improves the efficiency of product development.

The switching component of STEP is mainly composed of EXPRESS modeling tools, EXPRESS compiler, STEP data processor, application of protocol processor and the model mapping and other function modules. Most of the existing 3D CAD, CAE and CAM systems, such as UG, CATIA, ProE et al., are configured with STEP AP2O3 interface, which can be used to realize the model transformation of products the and integration between systems.

The Data Integration Technology.Data integration is the bridge of heterogeneous data mapping metadata model. Firstly, data source is figured out to connect the new data in business activity and the old data of legacy systems. And then a data integration mechanism is developed to realize data deposition in BLDMS from other heterogeneous systems via extraction, transformation and loading (ETL) [7] and extensive markup language (XML).

The ETL is a method to realize the integration of heterogeneous data. It is responsible for extracting the distributed heterogeneous data, such as relational data and graphic data files to the temporary middle layer, after cleaning, conversion and integration, and completing three operations that the correction of the data format, the merging of the data fields, as well as the new indicator.

$\mathrm{XML}$ is a way of heterogeneous data integration based on the schema mapping. It is needless to change the way of the original data storage and management. This paper utilizes Kettle, which is an open source tool, to effectively realize the process of ETL transformation. It is able to make all kinds of data acquisition and the output in a specified format. From the above, the data integration mechanism is shown in fig4. 


\section{Conclusion}

This paper mainly researches the overall design and key technology of BLDMS, and lays the foundation for the practical system development. The proposed BLDMS opens the mode of optimization management for numerical control machine tool bearings. The expectation of this system is that designers can use historical data to improve the design work; production processing sectors can detect the quality status of products; and machine tool users can detect the running state of the machine tool real-time and bearing maintenance timely. Hereby, the key technology is not only proposing method for the bearing information storage and retrieval efficiency, and also providing a solution to the information island problem in space and time for enterprises. Thus the process of data sharing will be completed and the utilization rate of the data will increase greatly.

\section{Acknowledgment}

This project is supported by the National Science \& Technology Major Program of China (No. 2012ZX04004011-05), Key scientific research projects in Universities of Henan Province (13B460000), and the Program for Innovative Research Team (in Science and Technology) in University of Henan Province (No. 15IRTSTHN008).

\section{References}

[1] YANG Xian-qi, LIU Zhong-xian, LI Zhan-bin. Bearing Enterprise PDM System[J]. Bearing, 2002(3): 32-35.

[2] MA Ming-xu, FAN Yun-shun, YI Chao-wan. Product Lifecycle Structural Model. Computer Integrated Manufacturing Systems, 2006, 12(8): 1153-1160.

[3] Kiviluoma, R.1, Pyykönen, et al. Load-bearing Assessment of Bridges by Iinternet-based Tools, 7th International Conference on Bridge Maintenance, Safety and Management, IABMAS,2014. Shanghai, China: Taylor and Francis Balkema,2014: 2367-2372.

[4] Freire, Luís-M.R, De-Brito, et al. Management System for Road Bridge Structural Bearings. Structure and Infrastructure Engineering, 2014,8(10):1068-1086.

[5] LI You-tan, LI Xiu-ling. Product Structure Model for Life-cycle Management. Journal of Lanzhou University of Technology, 2008, 34(4): 34-39.

[6] JIANG Zhan, YAO Xiao-ming, LAN Fen. Feature-based Adaptive Method of Ontology Mapping. Journal of Zhejiang University (Engineering Science),2014. 48(1):76-84.

[7] WANG Shi-shui, WANG Yuan-yuan, GAO Ying-bo. Research and realizations of data integration ETLbased optimization. Journal of Tianjin Polytechnic University, 2013,32(3):78-81. 